OPEN ACCESS

Edited by:

Susan Currie,

University of Strathclyde,

United Kingdom

Reviewed by:

Alessandra Cuomo,

Federico II University Hospital, Italy George Lazaros,

Hippokration General

Hospital, Greece

*Correspondence:

Masayoshi Oikawa

moikawa@fmu.ac.jp

Specialty section: This article was submitted to

Cardio-Oncology,

a section of the journal

Frontiers in Cardiovascular Medicine

Received: 14 August 2020 Accepted: 06 October 2020 Published: 30 October 2020

Citation:

Yaegashi D, Oikawa M, Yokokawa T, Misaka T, Kobayashi A, Kaneshiro T, Yoshihisa A, Nakazato K, Ishida T and Takeishi Y (2020) Red Blood Cell Distribution Width Is a Predictive Factor of Anthracycline-Induced Cardiotoxicity.

Front. Cardiovasc. Med. 7:594685.

doi: 10.3389/fcvm.2020.594685

\section{Red Blood Cell Distribution Width Is a Predictive Factor of Anthracycline-Induced Cardiotoxicity}

\author{
Daiki Yaegashi, Masayoshi Oikawa*, Tetsuro Yokokawa, Tomofumi Misaka, \\ Atsushi Kobayashi, Takashi Kaneshiro, Akiomi Yoshihisa, Kazuhiko Nakazato, \\ Takafumi Ishida and Yasuchika Takeishi
}

Department of Cardiovascular Medicine, Fukushima Medical University, Fukushima, Japan

Background: Red blood cell distribution width (RDW) is associated with prognosis in widespread cardiovascular fields, but little is known about relationship with the onset of cancer therapeutics-related cardiac dysfunction (CTRCD).

Objectives: The purpose of this study was to assess whether RDW could predict the onset of CTRCD by anthracycline.

Methods: Consequential 202 cancer patients planed for anthracycline treatment were enrolled and followed up for 12 months. The patients were divided into 2 groups based on the median value of baseline RDW before chemotherapy [low RDW group, $n=98$, 13.0 [12.6-13.2]; high RDW group, $n=104,14.9$ [13.9-17.0]]. Cardiac function was assessed serially by echocardiography at baseline (before chemotherapy), as well as at 3, 6, and 12 months after chemotherapy with anthracycline.

Results: Baseline left ventricular end systolic volume index and ejection fraction (EF) were similar between two groups. After chemotherapy, EF decreased at 3- and 6-month in the high RDW group [baseline, 64.5\% [61.9-68.9\%]; 3-month, 62.6\% [60.4-66.9\%]; 6-month, 63.9\% [60.0-67.9\%]; 12-month, 64.7\% [60.8-67.0\%], $P=0.04$ ], but no change was observed in low RDW group. The occurrence of CTRCD was higher in high RDW group than in low RDW group (11.5 vs. $2.0 \%, P=0.008)$. When we set the cut-off value of RDW at 13.8, sensitivity and specificity to predict CTRCD were 84.6 and $62.0 \%$, respectively. Multivariable logistic regression analysis revealed that baseline RDW value was an independent predictor of the development of CTRCD [odds ratio 1.390, 95\% Cl [1.09-1.78], $P=0.008$ ]. The value of net reclassification index (NRI) and integrated discrimination improvement (IDI) for detecting CTRCD reached statistical significance when baseline RDW value was added to the regression model including known risk factors such as cumulative anthracycline dose, EF, albumin, and the presence of hypertension; $0.9252(95 \% \mathrm{Cl} 0.4103-1.4402, P<0.001)$ for $\mathrm{NRI}$ and $0.1125(95 \% \mathrm{Cl}$ $0.0078-0.2171, P=0.035)$ for IDI.

Conclusions: Baseline RDW is a novel parameter to predict anthracycline-induced CTRCD.

Keywords: cardio-oncology, anthracycline, red blood cell distribution width, cancer therapeutics-related cardiac dysfunction, heart failure 2 


\section{INTRODUCTION}

Anthracycline-containing chemotherapy is highly effective and widely used in cancer treatments. However, anthracyclineinduced cardiotoxicity is associated with a poor prognosis in cancer survivors, and the frequency of onset depends on risk factors including cumulative dose of anthracycline, elderly or pediatric population, concomitant or previous radiation therapy, pre-existing cardiovascular diseases, and concomitant use of human epidermal growth factor receptor 2 (HER2) inhibitors (1). Several hypotheses have been proposed for the mechanisms of anthracycline-induced cardiotoxicity, such as oxidative stress, iron accumulation in myocardial cells, mitochondrial dysfunction, and topoisomerase $2 \beta$ dysfunction (2-5). Most anthracycline-induced cardiotoxicity occurs within the first year of chemotherapy, and early detection and treatment may recover cardiac function (6). Therefore, assessment of cardiac function should be performed to detect early phase of cardiotoxicity, and accurate predictors are required to identify patients predisposed to anthracycline-induced cardiotoxicity.

Red blood cell distribution width (RDW) is a simple and rapid measurement of the heterogeneity of erythrocyte volume. Growing body of evidence suggests that high RDW is strongly associated with poor prognosis in widespread cardiovascular diseases such as acute coronary syndrome, heart failure, and pulmonary hypertension (7-9). However, the association between anthracycline-induced cardiotoxicity and RDW have not been rigorously examined. The purpose of the present study was to assess whether baseline RDW could predict the onset of cancer therapeutics-related cardiac dysfunction (CTRCD) by anthracycline-containing chemotherapy.

\section{METHODS}

\section{Study Subjects and Protocol}

We enrolled 234 consecutive cancer patients, planned for initial anthracycline-containing chemotherapy at Fukushima Medical University hospital from February 2017 to September 2019 (Figure 1). Patients were excluded if they were died or transferred to other hospitals within 12 months follow-up period $(n=32)$. Remaining 202 patients were divided into 2 groups based on the median value of RDW (13.6) before chemotherapy: low RDW group, $n=98,13.0$ [12.6-13.2] and high RDW group, $n=104$, 14.9 [13.9-17.0].

Hypertension was defined as a history of use of antihypertensive drug or systolic blood pressure of $\geq 140$ $\mathrm{mmHg}$, and/or diastolic blood pressure $\geq 90 \mathrm{mmHg}$. Diabetes was defined as a recent use of insulin treatment or hypoglycemic drug, or hemoglobin A1c $\geq 6.5 \%$. Dyslipidemia was defined as a history of use of cholesterol-lowering drugs, or triglyceride was $\geq 150 \mathrm{mg} / \mathrm{dl}$, low density lipoprotein cholesterol was $\geq 140 \mathrm{mg} / \mathrm{dl}$, and/or high-density lipoprotein cholesterol was $\leq 40 \mathrm{mg} / \mathrm{dl}$. Cumulative dose of anthracycline was expressed as a doxorubicin equivalent (1). HER 2 inhibitor included trastuzumab and pertuzumab. Radiation therapy was defined as irradiation to the mediastinum. Transthoracic echocardiography and blood sampling test were performed at baseline, as well as at
3, 6, and 12 months after administration of anthracyclines. All procedures used in this research were approved by the Ethical Committee of Fukushima Medical University Hospital.

\section{Echocardiography}

Transthoracic echocardiography was performed by a trained sonographer, and images were checked by another trained sonographer and an echo-cardiologist. We measured cardiac function using EPIQ 7G (Philips Healthtech, Best, Netherland). EF was calculated using the modified Simpson's method according to the guideline from the American Society of Echocardiography and the European Association of Cardiovascular Imaging (10). The left ventricular (LV) mass was calculated using the following formula:

\section{Left ventricular $(\mathrm{LV})$ mass}

$=0.8 \times[1.04 \times\{(\mathrm{LV}$ diastolic diameter + interventricular septum wall thicness $+\mathrm{LV}$ posterior wall thicness $)^{3}$

$\left.\left.-(\text { LV diastolic diameter })^{3}\right\}\right]+0.6 \mathrm{~g}[10]$.

CTRCD was defined as a decrease in EF more than $10 \%$ points, to a value $<53 \%$ (11). LV diastolic dimension index, LV systolic dimension index, LV end-diastolic volume index, LV end-systolic volume index, and left atrial volume index (LAVI) were measured using B-mode ultrasound.

\section{Blood Sampling}

Blood sampling was performed in a stable condition after an overnight fast. High sensitivity cardiac troponin I (TnI) was measured using an assay based on Luminescent Oxygen Channeling Immunoassay technology, and run on a Dimension EXL integrated chemistry system (Siemens Healthcare Diagnostics, Deerfield, IL, USA). B-type natriuretic peptide (BNP) levels were measured using a specific immunoradiometric assay (Shionoria BNP kit, Shionogi, Osaka, Japan). RDW was measured using a DxH800 (Beckman Coulter, Inc., Fullerton, CA, USA).

\section{Statistical Analysis}

All statistical analyzes were performed using SPSS version 26 (IBM, Armonk, New York, USA) or R software packages version 3.6.3 (R core team 2020, Vienna, Austria). We used the ShapiroWilk test to discriminate which variables were normally or not normally distributed. Normally distributed variables were shown as mean \pm standard deviation. Non-normally distributed variables were indicated by median with interquartile range. Category variables were shown in numbers and percentage. Student's $t$-test was used for variables following a normal distribution, the Mann-Whitney $U$-test was used for variables of the non-normal distribution, and the $\chi$-square test was used for categorical variables. The time course of EF (baseline, 3-, 6-, and 12-month after the administration of anthracyclines) was evaluated using the Friedman test.

Logistic regression analysis was performed to identify variables relating to the occurrence of CTRCD. We selected variables as follows: age, sex, hypertension, dyslipidemia, diabetes mellitus, current or past smoker, cumulative anthracycline dose, 


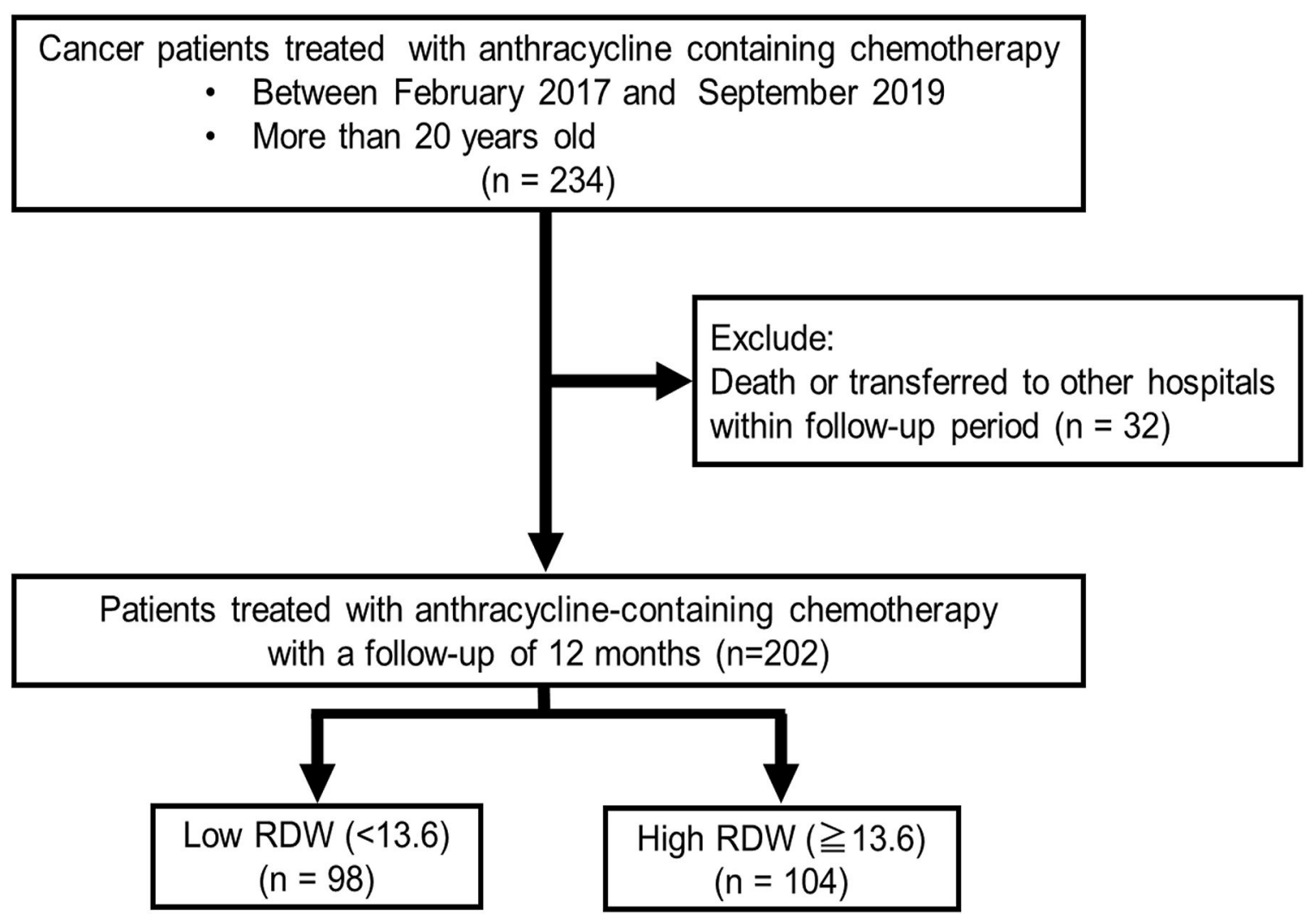

FIGURE 1 | Patient cohort selection.

use of HER2 inhibitor, BNP, albumin, LV mass index, LV end-diastolic volume index, LV end-systolic volume index, EF, LAVI, and RDW. Akaike Information Criteria was used for selecting variables for multivariate analysis. Receiver operator characteristic curve (ROC) analysis was performed to determine the optimal cut-off value of RDW for predicting the occurrence of CTRCD. The improvement by adding RDW to discrimination and net reclassification of risks was assessed by the estimation of both integrated discrimination improvement (IDI) and net reclassification improvement (NRI). The $P$-value of 0.05 or less was defined as significant.

\section{RESULTS}

Table 1 showed patient characteristics at the baseline of chemotherapy. The median value of RDW of all patients before chemotherapy was 13.6, and patients were divided into 2 groups based on the median value. Of 202 patients, 104 patients were grouped as high RDW group (13.6 and above), and 98 patients were grouped as low RDW group $(<13.6)$. There were no statistical differences in age, sex, coronary risk factors, cumulative anthracycline dose, the usage of HER2 inhibitor, and radiation therapy between the two groups. High RDW group included lower rate of breast cancer (60.2 vs. $43.2 \%, P=0.016$ ). Laboratory data showed that hemoglobin and mean corpuscular volume were lower in high RDW group, but BNP and TnI levels at baseline were similar between the two groups. The usage of angiotensin-converting enzyme (ACE) inhibitors, angiotensin II receptor blockers (ARB), $\beta$-blockers were similar between the two groups. Echocardiographic data demonstrated that LVEDVI, LVMI, and EF at baseline were similar between the two groups. Laboratory data showed similar liver and renal function.

Time-dependent changes in EF are displayed in Figure 2. EF decreased at 3- and 6-month after chemotherapy in high RDW group [baseline, 64.5\% [61.9-68.9\%]; 3-month, 62.6\% [60.466.9\%]; 6-month, 63.9\% [60.0-67.9\%]; 12-month, 64.7\% [60.8$67.0 \%], P=0.04$, Figure 2B], but no change was observed in low RDW group (Figure 2A). The occurrence of CTRCD during the 12-month follow-up period was higher in high RDW group than in low RDW group $[n=12[11.5 \%]$ vs. $n=2[2.0 \%] P=$ $0.008]$. When we set the cut-off value of RDW at 13.8 from the ROC analysis, sensitivity, specificity, and area under the curve to predict CTRCD were $84.6,62.0$, and $0.769 \%$, respectively, as shown in Figure 3. Multivariable logistic regression analysis revealed that RDW at baseline was an independent predictor of the development of CTRCD [odds ratio 1.390, 95\% CI [1.091.78], $P=0.008$; Table 2].

To assess the importance of adding RDW more precisely, NRI, and IDI were calculated using the variables with or without RDW. The value of NRI and IDI for detecting CTRCD reached 
TABLE 1 | Baseline clinical characteristics of patients with high or low RDW.

\begin{tabular}{|c|c|c|c|}
\hline & $\begin{array}{l}\text { Low RDW } \\
(n=98)\end{array}$ & $\begin{array}{l}\text { High RDW } \\
(n=104)\end{array}$ & $P$-value \\
\hline Age, years & $58.0(47.5-66.0)$ & $54.0(45.0-65.0)$ & 0.396 \\
\hline Female, n (\%) & $81(82.6)$ & $81(77.8)$ & 0.395 \\
\hline Body mass index, $\mathrm{kg} / \mathrm{m}^{2}$ & $22.9 \pm 3.7$ & $23.7 \pm 4.5$ & 0.161 \\
\hline \multicolumn{4}{|l|}{ Cardiovascular risk factor } \\
\hline Hypertension, n (\%) & $21(21.4)$ & $26(25.0)$ & 0.426 \\
\hline Dyslipidemia, n (\%) & $34(34.6)$ & $23(22.1)$ & 0.101 \\
\hline Diabetes mellitus, $n(\%)$ & $10(10.2)$ & $12(11.5)$ & 0.669 \\
\hline Current or past smokers, n (\%) & $33(33.6)$ & $36(34.6)$ & 0.849 \\
\hline Family history of CAD, n (\%) & $9(9.1)$ & $14(13.4)$ & 0.270 \\
\hline $\begin{array}{l}\text { CKD }\left(e G F R<60 \mathrm{ml} / \mathrm{min} / 1.73 \mathrm{~m}^{2}\right) \\
\mathrm{n}(\%)\end{array}$ & $15(15.3)$ & $15(14.4)$ & 0.858 \\
\hline \multicolumn{4}{|c|}{ Pre-treatment cardiovascular medications } \\
\hline Aspirin, n (\%) & $2(2.0)$ & $3(2.8)$ & 0.528 \\
\hline Statin, n (\%) & $9(9.1)$ & $10(9.6)$ & 0.916 \\
\hline$\beta$-blocker, n (\%) & $1(1.0)$ & $2(1.9)$ & 0.522 \\
\hline ACE inhibitor and/or ARB, $\mathrm{n}(\%)$ & $18(18.3)$ & $12(11.5)$ & 0.173 \\
\hline \multicolumn{4}{|l|}{ Anti-cancer treatment } \\
\hline $\begin{array}{l}\text { Cumulative anthracycline dose, } \\
\mathrm{mg} / \mathrm{m}^{2}\end{array}$ & $200(180-240)$ & $180(150-250)$ & 0.203 \\
\hline HER2 inhibitor, n (\%) & $15(15.3)$ & $10(9.6)$ & 0.220 \\
\hline Radiation therapy, n (\%) & $7(7.1)$ & $6(5.7)$ & 0.774 \\
\hline \multicolumn{4}{|l|}{ Oncological disease } \\
\hline Breast cancer, n (\%) & $59(60.2)$ & $45(43.2)$ & 0.016 \\
\hline Hematological tumor, n (\%) & 20 (20.4) & $33(31.7)$ & 0.068 \\
\hline Gynecologic tumor, n (\%) & $10(10.2)$ & $14(13.4)$ & 0.475 \\
\hline Osteosarcoma, n (\%) & $8(8.1)$ & $9(8.6)$ & 0.900 \\
\hline Other solid tumor, n (\%) & $1(1.0)$ & $3(2.8)$ & 0.333 \\
\hline \multicolumn{4}{|l|}{ Laboratory data } \\
\hline BNP, pg/ml & $11.2(6.6-19.8)$ & $12.4(5.9-22.7)$ & 0.665 \\
\hline Troponin I, ng/ml & $\begin{array}{c}0.017 \\
(0.017-0.017)\end{array}$ & $\begin{array}{c}0.017 \\
(0.017-0.017)\end{array}$ & 0.776 \\
\hline Total protein, g/dl & $7.10(6.8-7.5)$ & $7.0(6.7-7.4)$ & 0.187 \\
\hline Albumin, g/dl & $4.2(3.9-4.5)$ & $4.0(3.8-4.3)$ & 0.003 \\
\hline AST, U/L & $18(15-21)$ & $19(15-24)$ & 0.788 \\
\hline ALT, U/L & $15(12-21)$ & $14(10-25)$ & 0.560 \\
\hline $\mathrm{LDH}, \mathrm{U} / \mathrm{L}$ & $181(153-215)$ & $197(165-244)$ & 0.060 \\
\hline BUN, mg/dl & $13.0(10.7-15.0)$ & $13.0(10.0-15.0)$ & 0.509 \\
\hline Creatinine, mg/dl & $0.65(0.57-0.76)$ & $0.66(0.57-0.78)$ & 0.588 \\
\hline eGFR, $\mathrm{ml} / \mathrm{min} / 1.73 \mathrm{~m}^{2}$ & $77.0(63.7-89.2)$ & $72.0(65.0-89.0)$ & 0.705 \\
\hline Total cholesterol, mg/dl & $201.9 \pm 36.9$ & $191.5 \pm 42.6$ & 0.099 \\
\hline Triglyceride, mg/dl & $\begin{array}{c}106 \\
(68.2-166.7)\end{array}$ & $\begin{array}{c}107.5 \\
(79.7-227.5)\end{array}$ & 0.979 \\
\hline $\begin{array}{l}\text { High density lipoprotein cholesterol, } \\
\mathrm{mg} / \mathrm{dl}\end{array}$ & $54.9 \pm 15.0$ & $53.1 \pm 15.5$ & 0.458 \\
\hline $\begin{array}{l}\text { Low density lipoprotein cholesterol, } \\
\mathrm{mg} / \mathrm{dl}\end{array}$ & $120.7 \pm 30.9$ & $113.2 \pm 36.4$ & 0.157 \\
\hline Hemoglobin A1c, \% & $5.6(5.4-5.8)$ & $5.7(5.5-6.1)$ & 0.185 \\
\hline C-reactive protein, mg/dl & $0.11(0.04-0.38)$ & $0.16(0.07-0.70)$ & 0.024 \\
\hline Uric acid, mg/dl & $4.3(3.4-5.3)$ & $4.7(3.6-5.3)$ & 0.876 \\
\hline D dimer, $\mu \mathrm{g} / \mathrm{ml}$ & $0.5(0.5-0.925)$ & $0.9(0.5-2.3)$ & $<0.001$ \\
\hline
\end{tabular}

(Continued)
TABLE 1 | Continued

\begin{tabular}{|c|c|c|c|}
\hline & $\begin{array}{l}\text { Low RDW } \\
(n=98)\end{array}$ & $\begin{array}{l}\text { High RDW } \\
(n=104)\end{array}$ & $P$-value \\
\hline White blood cell, $\mu \mathrm{l}$ & $\begin{array}{c}5,550 \\
(4,450-6,800)\end{array}$ & $\begin{array}{c}5,500 \\
(4,200-7,200)\end{array}$ & 0.958 \\
\hline Hemoglobin, g/dl & $13.1(12.4-13.8)$ & $11.8(10.5-13.2)$ & $<0.001$ \\
\hline $\mathrm{MCV}, \mathrm{fl}$ & $93.0 \pm 4.2$ & $89.2 \pm 8.9$ & $<0.001$ \\
\hline $\mathrm{PLT}, \times 10^{3} / \mu \mathrm{l}$ & $252.5 \pm 72.8$ & $246.6 \pm 96.1$ & 0.624 \\
\hline RDW & $13.0(12.6-13.2)$ & $14.9(13.9-17.0)$ & $<0.001$ \\
\hline \multicolumn{4}{|l|}{ Echocardiographic data } \\
\hline LVDdl, mm/m² & $26.6(24.5-29.2)$ & $26.3(24.5-28.5)$ & 0.604 \\
\hline LVDsl, mm/m² & $16.2 \pm 2.4$ & $16.4 \pm 2.8$ & 0.680 \\
\hline LVMI, $g / \mathrm{m}^{2}$ & $67.8(58.7-82.1)$ & $71.3(61.8-84.9)$ & 0.076 \\
\hline LVEDVI, ml/m² & $47.0 \pm 13.6$ & $46.6 \pm 18.6$ & 0.844 \\
\hline LVESVI, ml/m² & $16.1(13.4-20.9)$ & $16.2(12.4-20.1)$ & 0.668 \\
\hline$E F, \%$ & $64.1 \pm 4.9$ & $65.1 \pm 5.2$ & 0.181 \\
\hline LAVI, ml/m² & $22.8(17.1-28.4)$ & $22.9(17.3-31.5)$ & 0.333 \\
\hline E/A & $0.97(0.75-0.20)$ & $1.01(0.76-1.19)$ & 0.710 \\
\hline $\begin{array}{l}\text { Mitral regurgitation (mild and above) } \\
\mathrm{n},(\%)\end{array}$ & $20(20.2)$ & $17(16.5)$ & 0.793 \\
\hline $\begin{array}{l}\text { Aortic regurgitation (mild and above) } \\
\mathrm{n},(\%)\end{array}$ & $7(7.1)$ & $8(7.8)$ & 0.850 \\
\hline $\begin{array}{l}\text { Aortic stenosis (mild and above) } \mathrm{n} \text {, } \\
(\%)\end{array}$ & 0 & $2(1.9)$ & 0.259 \\
\hline TR-PG, mmHg & $19.3(16.2-22.3)$ & $19.0(15.0-23.0)$ & 0.463 \\
\hline RVD, mm & $27.2 \pm 6.3$ & $28.7 \pm 5.6$ & 0.098 \\
\hline
\end{tabular}

Values are indicated by mean $\pm S D$, median with interquartile range or $n(\%)$. Monoclonal antibodies include HER2 inhibitor. Radiation therapy includes only irradiation to the mediastinum. Hemato-oncologic disease include Hodgkin disease, Non-Hodgkin disease, acute myeloid leukemia, acute lymphocytic leukemia, and chronic lymphocytic leukemia. Gynecologic tumor include uterine cancer or sarcoma and ovarian cancer. Other solid tumors include thymoma, soft tissue tumor, teratoma.

$A C E$, angiotensin-converting enzyme; $A R B$, angiotensin II receptor blocker; $B N P, B$ type natriuretic peptide; CAD, coronary artery disease; CKD, chronic kidney disease; eGFR, estimated glomerular filtration rate; GLS, global longitudinal strain; HER2, human epidermal growth factor receptor 2; LAVI, left atrial volume index; LV, left ventricular; LVDdl, LV diastolic dimension index; LVDsI, LV systolic dimension index; LVEDVI, LV end-diastolic volume index; EF, ejection fraction; LVESVI, LV end-systolic volume index; LVMI, LV mass index; MCV, mean corpuscular volume; RVD, right ventricular dimension; TR-PG, tricuspid pressure gradient.

statistical significance when baseline RDW value was added to the model including cumulative dose of anthracycline, EF, albumin and hypertension; 0.9252 (95\%CI $0.4103-1.4402, P<0.001)$ for NRI and 0.1125 (95\%CI $0.0078-0.2171, P=0.035)$ for IDI.

\section{DISCUSSION}

In the present study, we revealed the clinical features of RDW in patients treated with anthracycline. First, EF was temporary decreased in high RDW patients. Second, the occurrence of anthracycline-induced CTRCD was significantly higher in high RDW patients. Third, high RDW at baseline was an independent predictor of the development of CTRCD.

Oxidative stress, iron accumulation in myocardial cells, mitochondrial dysfunction, and topoisomerase $2 \beta$ dysfunction 
A

Low RDW

$\mathrm{P}=0.990$

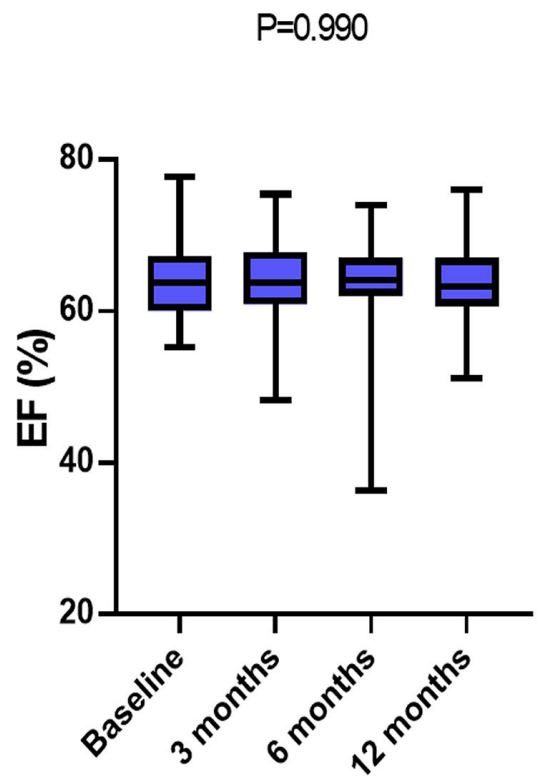

B

\section{High RDW}

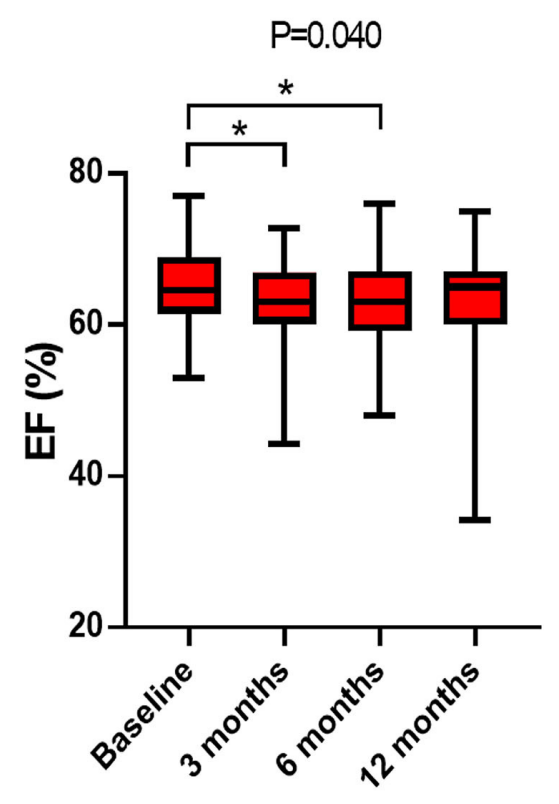

FIGURE 2 | Time-dependent changes in EF after anthracycline treatment. (A) Changes in EF in low RDW group. (B) Changes in EF in high RDW group. ${ }^{\star} P<0.05$.

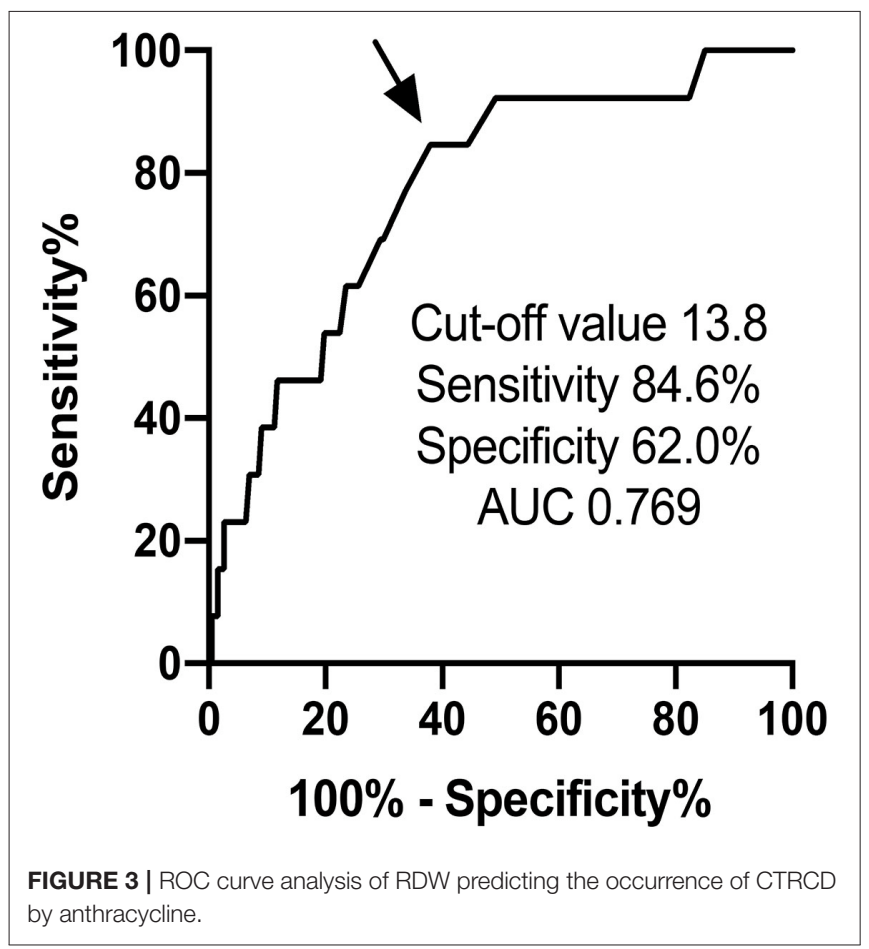

have been proposed as major mechanisms of anthracyclineinduced cardiotoxicity $(2-5)$. The excess production of free radicals by the quinone group from aglycons of anthracycline induces oxidation of protein, nucleic acid, and lipid, leading to cellular damage (4). It has been reported that elevation of RDW is also associated with oxidative stress as well as aging, inflammation, malnutrition, and renal dysfunction $(12,13)$. Among those factors, oxidative stress induces bone marrow dysfunction, leading to abnormal heme synthesis, and hemoglobin production that increased in RDW. In the present study, the patients with high RDW at baseline demonstrated time-dependent decrease in EF and higher occurrence of CTRCD. These findings raise the possibility that the patients with high RDW already had been exposed to increased oxidative stress before chemotherapy, and were more vulnerable to additional oxidative stress by anthracycline, resulting in the development of CTRCD.

Although underlying mechanisms of interaction between RDW and CTRCD remains to be elucidated, to the best of our knowledge, this is the first report to demonstrate the association between RDW and CTRCD.

The utility of RDW has been reported in not only cardiovascular disease, but also cancer prognosis. High RDW are associated with unfavorable survival in many types of cancers including breast cancer (14), hematological tumor (15), gynecological cancer $(16,17)$, and osteosarcoma (18). Although there is no clear explanation of the relationship between RDW and cancer prognosis, RDW can be a promising biomarker for assessment of the risk of CTRCD and cancer prognosis. Cardinale et al. (6) reported that $78 \%$ patients with anthracycline-induced cardiotoxicity showed a full or partial recovery with heart failure therapy. In this cohort, one patient presented $<40 \%$ of ejection fraction at 6-month follow-up. He was administered $\beta$-blockers and ACE inhibitors soon after the onset of CTRCD. Then, his 
TABLE 2 | Parameters associated with the occurrence of CTRCD.

\begin{tabular}{|c|c|c|c|c|}
\hline & \multicolumn{2}{|c|}{ Univariable } & \multicolumn{2}{|c|}{ Multivariable* } \\
\hline & Odds Ratio $(95 \% \mathrm{Cl})$ & $P$-value & Odds Ratio (95\% Cl) & $P$-value \\
\hline Age, per 1-year increase & $0.986(0.950-1.023)$ & 0.455 & & \\
\hline Female & $0.898(0.239-0.384)$ & 0.874 & & \\
\hline Hypertension & $0.511(0.110-2.371)$ & 0.391 & $0.264(0.03-1.78)$ & 0.171 \\
\hline Dyslipidemia & $0.186(0.024-1.446)$ & 0.110 & & \\
\hline Diabetes mellitus & $0.586(0.073-4.711)$ & 0.615 & & \\
\hline Current or past smoker & $0.299(0.065-1.374)$ & 0.121 & & \\
\hline Cumulative anthracycline dose, $\mathrm{mg} / \mathrm{m}^{2}$ per 1.0 increase & $1.010(1.004-1.016)$ & 0.001 & $1.010(1.00-1.02)$ & $<0.001$ \\
\hline HER2 inhibitor & $0.526(0.066-4.201)$ & 0.544 & & \\
\hline Radiation therapy & $1.077(0.130-8.941)$ & 0.945 & & \\
\hline BNP, pg/ml per 1.0 increase & $1.000(0.976-1.025)$ & 0.998 & & \\
\hline Albumin, per 1.0 increase & 0.469 (0.171-1.286) & 0.141 & $0.367(0.09-1.35)$ & 0.131 \\
\hline LVMI, g/m² per 1.0 increase & $0.998(0.970-1.027)$ & 0.876 & & \\
\hline LVEDVI, $\mathrm{ml} / \mathrm{m}^{2}$ per 1.0 increase & $0.994(0.959-1.024)$ & 0.574 & & \\
\hline LVESVI, $\mathrm{ml} / \mathrm{m}^{2}$ per 1.0 increase & $1.072(0.970-1.178)$ & 0.149 & & \\
\hline EF, per 1.0 increase & $0.926(0.826-1.038)$ & 0.186 & $0.868(0.75-1.01)$ & 0.058 \\
\hline LAVI, ml/m² per 1.0 increase & 0.999 (0.967-1.031) & 0.824 & & \\
\hline RDW, per 1.0 increase & $1.378(1.126-1.687)$ & 0.002 & $1.390(1.09-1.78)$ & 0.008 \\
\hline
\end{tabular}

*adjusted for hypertension, cumulative anthracycline dose, albumin, ejection fraction, and RDW categorical variables.

$\mathrm{Cl}$, Confidence interval; CTRCD, cancer therapeutics-related myocardial disorder; RDW, red blood cell distribution width.

ejection fraction recovered to over $50 \%$ at 12 -month follow-up. We speculate that early initiation of cardioprotective therapy induced a recovery of cardiac function. Thus, early detection and treatments of CTRCD are essential to minimize cardiac damage induced by anthracyclines. TnI and BNP are widely used surrogate biomarkers to detect CTRCD, but the elevation occurs after starting anthracycline chemotherapy (19). Compared to them, RDW can predict the development of CTRCD before chemotherapy. By assessing RDW before chemotherapy, precise follow-up management can be scheduled, and indication of prophylaxis treatment can be discussed. To date, several clinical studies have demonstrated that angiotensin-converting enzyme and beta-adrenergic receptor blockers prevent cardiac dysfunction after anthracycline chemotherapy (20). According to previous reports of anti-oxidative effects of carvedilol and ACE inhibitor (21), the efficacy of prophylaxis may be expected to the patients with high RDW.

In the present study, the calculation of NRI, and IDI demonstrated the significance of RDW for the prediction of CTRCD. The utility of RDW should be considered when managing cancer patients treated with anthracyclinecontaining chemotherapy.

\section{LIMITATION}

This study was performed using a relatively small number of patients and short follow-up period by a single center. Longer follow-up and larger population data were needed to confirm the importance of RDW to the development of CTRCD and cardiovascular prognosis. According to 2016 ESC position paper (1), 3 dimensional-based LVEF have advantages to assess cardiac function compared to Simpson's method, and global longitudinal strain reveals subtle changes in left ventricular function, and thus detects myocardial damage in the early stage before reduces in left ventricular ejection fraction (22). Further studies using 3 dimentional echocardiography and global longitudinal strain are desirable in the future.

\section{CONCLUSION}

Baseline RDW identifies high risk patients with anthracyclineinduced CTRCD.

\section{DATA AVAILABILITY STATEMENT}

The raw data supporting the conclusions of this article will be made available by the authors, without undue reservation.

\section{ETHICS STATEMENT}

The studies involving human participants were reviewed and approved by Ethical Committee of Fukushima Medical University Hospital. The patients/participants provided their written informed consent to participate in this study.

\section{AUTHOR CONTRIBUTIONS}

MO conceived of the presented idea. MO and DY developed the theory and performed the computations. TY, TM, AK, TK, AY, and KN verified the analytical methods. YT and TI supervised the findings of this work. All authors discussed the results and contributed to the final manuscript. 


\section{FUNDING}

This work was supported by JSPS KAKENHI (Grant Number JP20K08493).

\section{REFERENCES}

1. Zamorano JL, Lancellotti P, Munoz DR, Aboyans V, Asteggiano R, Galderisi M, et al. 2016 ESC Position Paper on cancer treatments and cardiovascular toxicity developed under the auspices of the ESC Committee for Practice Guidelines: The Task Force for cancer treatments and cardiovascular toxicity of the European Society of Cardiology (ESC). Eur Heart J. (2016) 37:2768801. doi: 10.1093/eurheartj/ehw211

2. Horenstein MS, Vander Heide RS, L'Ecuyer TJ. Molecular basis of anthracycline-induced cardiotoxicity and its prevention. Mol Genet Metab. (2000) 71:436-44. doi: 10.1006/mgme.2000.3043

3. Link G, Tirosh R, Pinson A, Hershko C. Role of iron in the potentiation of anthracycline cardiotoxicity: identification of heart cell mitochondria as a major site of iron-anthracycline interaction. J Lab Clin Med. (1996) 127:2728. doi: 10.1016/S0022-2143(96)90095-5

4. Ichikawa Y, Ghanefar M, Bayeva M, Wu R, Khechaduri A, Naga Prasad SV, et al. Cardiotoxicity of doxorubicin is mediated through mitochondrial iron accumulation. J Clin Invest. (2014) 124:617-30. doi: 10.1172/JCI72931

5. Zhang S, Liu X, Bawa-Khalfe T, Lu LS, Lyu YL, Liu LF, et al. Identification of the molecular basis of doxorubicin-induced cardiotoxicity. Nat Med. (2012) 18:1639-42. doi: 10.1038/nm.2919

6. Cardinale D, Colombo A, Bacchiani G, Tedeschi I, Meroni CA, Veglia F, et al. Early detection of anthracycline cardiotoxicity and improvement with heart failure therapy. Circulation. (2015) 131:1981-8. doi: 10.1161/CIRCULATIONAHA.114.013777

7. Abrahan LL, Ramos JDA, Cunanan EL, Tiongson MDA, Punzalan FER. Red cell distribution width and mortality in patients with acute coronary syndrome: a meta-analysis on prognosis. Cardiol Res. (2018) 9:14452. doi: 10.14740/cr732w

8. Felker GM, Allen LA, Pocock SJ, Shaw LK, McMurray JJ, Pfeffer MA, et al. Red cell distribution width as a novel prognostic marker in heart failure: data from the CHARM Program and the Duke Databank. J Am Coll Cardiol. (2007) 50:40-7. doi: 10.1016/j.jacc.2007.02.067

9. Hampole CV, Mehrotra AK, Thenappan T, Gomberg-Maitland M, Shah SJ. Usefulness of red cell distribution width as a prognostic marker in pulmonary hypertension. Am J Cardiol. (2009) 104:868-72. doi: 10.1016/j.amjcard.2009.05.016

10. Lang RM, Badano LP, Mor-Avi V, Afilalo J, Armstrong A, Ernande L, et al. Recommendations for cardiac chamber quantification by echocardiography in adults: an update from the American society of echocardiography and the European association of cardiovascular imaging. J Am Soc Echocardiogr. (2015) 28:1-39.e14. doi: 10.1016/j.echo.2014.10.003

11. Plana JC, Gaiderisi M, Barac A, Ewer MS, Bonnie Ky, Scherrer-Crosbie $\mathrm{M}$, et al. Expert consensus for multimodality imaging evaluation of adult patients during and after cancer therapy: a report from the American society of echocardiography and the European association of cardiovascular imaging. J Am Soc Echocardiogr. (2014) 27:911-39. doi: 10.1016/j.echo.2014. 07.012

12. salvagno gl, sanchis-gomar f, picanza A, Lippi G. Red blood cell distribution width: a simple parameter with multiple clinical applications.

\section{ACKNOWLEDGMENTS}

The authors thank for Ms. H. Kobayashi for data management.

Crit Rev Clin Lab Sci. (2015) 52:86-105. doi: 10.3109/10408363.2014.9 92064

13. Lippi G, Cervellin G, Sanchis-Gomar F. Red blood cell distribution width and cardiovascular disorders. Does it really matter which comes first, the chicken or the egg? Int J Cardiol. (2016) 206:129-30. doi: 10.1016/j.ijcard.2016.01.122

14. Yao D, Wang Z, Cai H, Li Y, Li B. Relationship between red cell distribution width and prognosis in patients with breast cancer after operation: a retrospective cohort study. Biosci Rep. (2019) 39:BSR20190740. doi: 10.1042/BSR20190740

15. Podhorecka M, Halicka D, Szymczyk A, Macheta A, Chocholska S, Hus $\mathrm{M}$, et al. Assessment of red blood cell distribution width as a prognostic marker in chronic lymphocytic leukemia. Oncotarget. (2016) 7:3284653. doi: 10.18632/oncotarget.9055

16. Li Z, Hong N, Robertson M, Wang C, Jiang G. Preoperative red cell distribution width and neutrophil-to-lymphocyte ratio predict survival in patients with epithelial ovarian cancer. Sci Rep. (2017) 7:43001. doi: $10.1038 /$ srep43001

17. Kemal Y, Demirag G, Baş B, Önem S, Teker F, Yücel I. The value of red blood cell distribution width in endometrial cancer. Clin Chem Lab Med. (2015) 53:823-7. doi: 10.1515/cclm-2014-0699

18. Zheng J, Yuan X, Guo W. Relationship between red cell distribution width and prognosis of patients with osteosarcoma. Biosci Rep. (2019) 39:BSR20192590. doi: 10.1042/BSR20192590

19. Oikawa M, Yoshihisa A, Yokokawa T, Misaka T, Yaegashi D, Miyata $\mathrm{M}$, et al. Cardiac troponin I predicts elevated B-type natriuretic peptide in patients treated with anthracycline-containing chemotherapy. Oncology. (2020) 98:653-60. doi: 10.1159/000507585

20. Blanter JB, Frishman WH. The preventive role of angiotensin converting enzyme inhibitors/angiotensin-ii receptor blockers and $\beta$-adrenergic blockers in anthracycline- and trastuzumab-induced cardiotoxicity. Cardiol Rev. (2019) 27:256-59. doi: 10.1097/CRD.0000000000000252

21. Chin BS, Langford NJ, Nuttall SL, Gibbs CR, Blann AD, Lip GY. Antioxidative properties of beta-blockers and angiotensin-converting enzyme inhibitors in congestive heart failure. Eur J Heart Fail. (2003) 5:1714. doi: 10.1016/S1388-9842(02)00251-9

22. Berliner D, Beutel G, Bauersachs J. Echocardiography and biomarkers for the diagnosis of cardiotoxicity. Herz. (2020). doi: 10.1007/s00059-020-04957-5. [Epub ahead of print].

Conflict of Interest: The authors declare that the research was conducted in the absence of any commercial or financial relationships that could be construed as a potential conflict of interest.

Copyright (C) 2020 Yaegashi, Oikawa, Yokokawa, Misaka, Kobayashi, Kaneshiro, Yoshihisa, Nakazato, Ishida and Takeishi. This is an open-access article distributed under the terms of the Creative Commons Attribution License (CC BY). The use, distribution or reproduction in other forums is permitted, provided the original author(s) and the copyright owner(s) are credited and that the original publication in this journal is cited, in accordance with accepted academic practice. No use, distribution or reproduction is permitted which does not comply with these terms. 